\title{
Städtische Kulturen und Bewegungen
}

Marcel Vejmelka

\section{Die Widersprüchlichkeit der brasilianischen Großstädte}

Im Vorfeld des brasilianischen Gastauftritts auf der Frankfurter Buchmesse 2013, der Fußballweltmeisterschaft 2014 in Brasilien und der Olympischen Sommerspiele 2016 in Rio de Janeiro erfährt auch die urbane Szenerie des Landes ein gesteigertes Medieninteresse. Doch bestimmte Klischeevorstellungen in Bezug auf Brasilien setzen sich auch in der zweiten Dekade des 21. Jahrhunderts fort und lassen sich nur schwer überwinden. Die aktuelle brasilianische Literatur erfährt innerhalb des internationalen Literaturbetriebs kaum Aufmerksamkeit und hat es dort selbst unter Experten schwer, sich von 'landestypischen' Erwartungshaltungen zu befreien. Die Medien widmen sich im Hinblick auf die beiden sportlichen Megaevents der kommenden Jahre vorrangig Themen wie den infrastrukturellen Vorbereitungen und stadtplanerischen Großprojekten, und berichten dabei mit Vorliebe über die diesen Maßnahmen zugrundeliegenden Probleme der Gewalt und Kriminalität in den Großstädten.

Dieser fast schon klassische Mediendiskurs über die extreme soziale Ungleichheit und die daraus resultierenden Spannungen im Stadtraum, die dort herrschende allgemeine Kriminalität und Gewalt, den organisierten Drogenkomplex und seine bewaffneten Auseinandersetzungen mit Polizei und Militär wird in den letzten Jahren um den Aspekt der seitens des Staates entwickelten Strategie zur Deeskalation dieses Dauerkonflikts erweitert. Besonderes Aufsehen und Diskussionen erregten dabei die zunächst in Rio de Janeiro zum Einsatz gebrachten Einheiten der "Friedenspolizei" (Unidade de Polícia Pacificadora, UPP).

Diese Entwicklungen schließen an eine über Jahrhunderte zurück reichende Geschichte an, in der Brasilien seit der Erschließung und Kolonisierung dieses riesigen Landes durch die Portugiesen von Städten, Stadträumen und Stadtkulturen geprägt wurde. Ein herausstechendes Merkmal dieser Geschichte ist bis heute der hohe Anteil der städtischen Bevölkerung - laut dem Zensus von 2010 leben aktuell ca. $84 \%$ der Brasilianer in Städten - und die übermächtige Bedeutung der urbanen Zentren, die in 
starkem Kontrast zu den kontinentalen Ausmaßen und dünn besiedelten Flächen des Landes stehen.

Während der ersten Jahrhunderte der portugiesischen Kolonialherrschaft bestand kaum Interesse oder Initiative hinsichtlich einer Besiedlung des Landesinnern, und die Reihe von Städten und Siedlungen an der Küste erfüllte vorrangig strategische Funktionen als Faktoreien, Festungen und Häfen. Erst mit der Entdeckung der riesigen Gold- und Edelsteinvorkommen und der sich daran anschließenden Einrichtung großflächiger Land- und Viehwirtschaft folgten auch verstärkte Gründungen strategisch situierter Städte im Landesinnern. Die gesellschaftlichen und kulturellen Wurzeln Brasiliens bildeten sich in diesen Küstenstädten heraus, die quantitativ immer weiter zunehmenden städtischen Vorposten im Landesinnern wirkten als Speerspitzen dieser urban geprägten 'Zivilisation', die sich daran machte, die unermesslichen Naturräume des Landes zu erschließen.

Die besondere Bedeutung der Städte in dieser historischen Entwicklung ist auch ablesbar an dem Umstand, dass Brasilien zweimal seine Hauptstadt verlegte. Der erste Sitz der Kolonialregierung in Salvador da Bahia wurde angesichts der Verlagerung des ökonomischen Schwerpunkts in die Gold- und Diamantengebiete weiter im Süden 1763 nach Rio de Janeiro verlegt, das sich zum wichtigsten Ausfuhrhafen für diese Werte entwickelte. Die bereits Ende des 19. Jahrhunderts getroffene Entscheidung, Brasilien administrativ wie auch symbolisch als moderne Nation mit einer neuen Hauptstadt zu gestalten, fand ihre endgültige Umsetzung 1960 mit der Einweihung des neu erbauten Brasília. Die von Lúcio Costa und Oscar Niemeyer nach modernistischen Grundsätzen konzipierte Modellstadt wurde bewusst ins geographische und symbolische Zentrum des Landes gesetzt, ihre innere Struktur - das Zentrum der politischen Macht, der berühmte plano piloto - ist durchweg funktional strukturiert und besitzt die Form eines Flugzeugs.

Insgesamt wird in diesem 'Wandern' der Hauptstadt und den jeweiligen Phasen strategischer Stadtgründungen sichtbar, wie in Brasilien durchgehend über die Gestaltung des Stadtraums der geografische Raum wie auch der gesellschaftliche Körper rational geordnet werden sollten. Bereits Ende des 19. Jahrhunderts beginnt so eine Reihe von strategisch geplanten und systematisch umgesetzten Stadtgründungen. Das dem Zukunftsprojekt Brasília vorangehende Jahrhundert war durchzogen von ähnlich funktionalistischen Stadtgründungen zur Erschließung des Landesinnern, so z. B. 1852 Teresina als Hauptstadt für den Bundesstaat Piauí, 1882 Rio 
Branco im Bundesstaat Acre, 1890 Boa Vista in Roraima im äußersten Norden, 1897 die Umgestaltung und Neugründung von Belo Horizonte als neue Hauptstadt von Minas Gerais, im 20. Jahrhundert dann 1933 Goiânia als Hauptstadt für den Bundesstaat Goiás und Palmas als Hauptstadt des kurz zuvor selbst erst gegründeten Bundesstaats Tocantins.

Diesen eine abstrakte Ordnung abbildenden Stadtentwürfen steht ebenfalls durch die gesamte brasilianische Geschichte hindurch die Realität der Großstädte gegenüber. Die Sklaverei hatte zunächst ihren Schwerpunkt auf den Plantagen und in den Minen im Landesinnern, doch spätestens mit ihrer Abschaffung Ende des 19. Jahrhunderts zogen ehemalige Sklaven wie auch andere Teile der verarmten Landbevölkerung massiv in die Städte. Dort lebten sie in armen Vorstädten oder illegalen Siedlungen, den Urformen der heutigen Favelas. Diese extreme sozio-ökonomische Segregation nahm schnell politisch wie administrativ nicht mehr kontrollierbare Ausmaße an, die urbanen Peripherien ließen eine bis heute prägende Dualität zwischen bürgerlicher und informeller Stadt entstehen. Zum Ende des 20. Jahrhunderts machte der Begriff der "Brasilianisierung" von Städten weltweit Karriere. Neben zugespitzten sozialen Konflikten im Stadtraum bezeichnete er auch die zunehmende Kommerzialisierung und Privatisierung des öffentlichen Raums. So verbindet man in der internationalen Wahrnehmung die brasilianischen Großstädte traditionell mit dem Begriff der "Krise", mit Problemen wie Armut, Kriminalität und Gewalt, einer zunehmenden Fragmentierung der urbanen Gesellschaft und ihrer Territorien.

Es ist ein historisches Paradox, dass die politische und soziale Elite Brasiliens ihren entschlossenen und kohärenten Willen zur Ordnung des nationalen Raums über strategische Stadtgründungen durch die interne Vernachlässigung dieser Stadträume ebenso konsequent selbst untergrub. Infrastrukturell durchdachte Stadtplanung wurde nicht betrieben, die armen Teile der Stadtbevölkerung wurden in der Regel einfach marginalisiert - an die Peripherien verdrängt und von der bürgerlichen Stadt ausgeschlossen - und das enorme und rasante Anwachsen dieser peripheren Stadträume beeinträchtigte unweigerlich die gesamte Lebensqualität und Bewohnbarkeit der Großstädte. Der Geograph Milton Santos beschrieb diese historisch bedingte Negativentwicklung in den 1990er Jahren mit dem Begriff des "Metropolenschwunds" (involução metropolitana).

Besonders problematisch dabei ist, dass der Druck der Peripherien auf die bürgerliche Stadt letztere dazu veranlasst, ihren eigenen urbanen Raum 
selbst zunehmend zu fragmentieren und privatisieren. Der bekannteste Faktor dieser Gegenbewegung ist die Entstehung räumlich abgegrenzter Enklaven für die gehobene Mittelschicht und die Oberschicht in Hochhauskomplexen (condominios verticais), geschlossenen Wohnanlagen (gated communities oder condominios fechados) oder auch außerhalb des Stadtgebiets in neuartigen metropolitanen Zentren, die nicht mehr der traditionellen Auffassung von Stadt entsprechen (Mega-Munizipien). Diese Stadtflucht der Reichen und die allgemeine Vernachlässigung der urbanen Peripherien verstärkten wie in einem Teufelskreis ihre Ursache: die Abwesenheit der staatlichen Macht in diesen armen Stadträumen, in deren Folge das dortige Machtvakuum ab den 1970er und vor allem 1980er Jahren zunehmend von der Drogenkriminalität ausgefüllt wurde.

Die armen Viertel und Favelas tragen aufgrund ihrer historischen Entstehung nach Abschaffung der Sklaverei und im Zuge der frühen Landflucht während der ersten Modernisierungsschübe um die Wende vom 19. zum 20. Jahrhundert das Stigma der Armut, des Elends, der Kriminalität und der systematischen Marginalisierung ihrer vorrangig afrobrasilianischen und farbigen Bevölkerung. Doch zeitgleich mit der allmählichen Verfestigung und Radikalisierung dieser Verschränkung von negativer Wahrnehmung und Realität bildet gerade dieser Raum die Keimzelle für kulturelle Formen und Manifestationen, ohne die Brasilien in seinem Selbstverständnis und erfolgreich verbreiteten Bild nicht denkbar wäre.

Mit den Scharen ehemaliger Sklaven und ihrer Nachkommen auf der Suche nach Arbeit und Überlebensmöglichkeiten füllten sich die teilweise zugewiesenen, teilweise durch Landbesetzungen entstandenen Armenviertel der Städte auch mit dem geistigen Erbe des Widerstands gegen die Sklaverei und den Frühformen eines (afro)brasilianischen Selbstbewusstseins. Die Quilombos - Siedlungen geflohener Sklaven - des 17. und 18. Jahrhunderts im Nordosten Brasiliens, in erster Linie der Quilombo de Palmares in Pernambuco, waren selbst befestigte Städte mit landwirtschaftlich genutztem Umland; die dramatischste und bekannteste messianische Bewegung der Region um Antônio Conselheiro hatte ihr Zentrum in der 1883 gegründeten Siedlung Canudos, die bei ihrer Zerstörung durch das Militär 1897 urbanen Charakter angenommen hatte. Beide Pole, die von der Kolonialmacht und der jungen Republik als existentielle Bedrohungen bekämpft und schließlich zerstört wurden, wirken in den modernen Stadtbereichen der Armut und des Elends bis heute fort. Gleichzeitig stellen sie in nicht geringerem Maße dynamische Räume dar, die hier nicht idealisiert 
oder verklärt werden dürfen, aber in ihrer Bedeutung für die Konfigurationen und Rekonfigurationen populärer Kultur im heutigen Brasilien wahrgenommen werden müssen. Und auch diese Bedeutung der aus der Sicht der bürgerlichen Stadt - des asfalto - als problematisch und bedrohlich empfundenen Bereiche der Armut und Informalität besitzt eine historische Dimension und eigene Tradition, die in jüngerer Vergangenheit zunehmende Anerkennung erfährt.

Die hier skizzierte Widersprüchlichkeit der brasilianischen Großstädte verdichtet sich somit in der einzigartigen Verbindung aus Alltagskultur und zivilgesellschaftlicher Selbstorganisation, die heute exemplarisch für das kreative Potenzial der Favelas und armen Vorstädte steht. Diese Verbindung, in der z. B. Musik zu einem entscheidenden Instrument politischer Repräsentation wurde, soll auf den folgenden Seiten anhand einiger Beispiele für brasilianische Stadtkulturen vorgestellt werden.

\section{Salvador und das afrobrasilianische Selbstbewusstsein}

Salvador da Bahia war bis 1763 erste Hauptstadt der Kolonie Brasilien und wichtigster Umschlagplatz für die aus Afrika verschleppten Sklaven, die vorrangig in der Plantagenwirtschaft eingesetzt wurden. Deren starke Präsenz über das Ende der Sklaverei hinaus prägte die Stadt entscheidend. So wird Salvador heute als das "schwarze Herz Brasiliens" bezeichnet und bildet zugleich das Zentrum des zum Ende des 20. Jahrhunderts etablierten afrobrasilianischen Selbstbewusstseins. Der von afrobrasilianischen Kulturformen durchdrungene Karneval von Salvador hat im Hinblick auf Größe und Bedeutung schon lange mit dem klassischen Modell aus Rio de Janeiro gleichgezogen und ist heute ein Magnet für Besucher aus der ganzen Welt. Es ist kein Zufall, dass die diesen Karneval gestaltenden Organisationen und Gruppen eine zentrale Bedeutung für das Erstarken der afrobrasilianischen Kultur in Bahia und ganz Brasilien haben.

Unter dem Einfluss der US-amerikanischen Bürgerrechtsbewegungen und globaler Diskurse um afrikanisches Erbe (négritude, Panafrikanismus) entstanden in den 1970er und 80er Jahren die blocos afro und betrieben im Rückgriff auf die lebendigen - stark religiös ausgerichteten - Traditionen des afoxé eine 'Afrikanisierung' des Karnevals von Salvador. Das bis dahin nur lokal und regional bedeutsame Ereignis wurde durch diese Neuerung aufgewertet und diente seinerseits als wichtige Plattform und Bühne, um 
die von den blocos repräsentierte Populärkultur sichtbar zu machen. So entstanden aus den Initiativen im unmittelbaren Kontext des Karnevals zahlreiche afrobrasilianische Kultur- und Sozialprojekte sowie Nichtregierungsorganisationen (NROs), die den erneuerten und kritischen Karneval mit ethno-politischem Aktivismus auch außerhalb dieser Sphäre verbanden.

Ein afoxé mit politischem und sozialem Engagement sind die Filhos de Gandhy, die bereits seit 1949 bestehen und sich besonders um die Wiederbelebung und Anerkennung der afrobrasilianischen Spiritualität verdient gemacht haben. $\mathrm{Zu}$ den erfolgreichsten und international bekanntesten Projekten zählen die blocos afro der ersten Stunde wie Ilê Ayê (1974 gegründet), die Grupo Cultural Olodum (1979) und Ara Ketu (1980). Olodum entstand 1979 als bloco afro und bemüht sich seit 1983 als NRO um die Bildung einer afrobrasilianischen kulturellen Identität, Antirassismus und black entrepreneurship. In diesem Rahmen wurden umfassende kulturelle und soziale Aktivitäten wie Theater, Tanz und Schulen entwickelt (<http://olodum.uol.com.br $>$ ). Das über Karneval, Musik und weitere kulturelle Angebote artikulierte Engagement dieser blocos afro und afoxés bewirkte gegen anfängliche Widerstände der Eliten eine zunehmende Integration des afrobrasilianischen Erbes in das Selbstbild Salvadors und Bahias. Im Stadtraum fand diese Entwicklung Anfang der 1990er Jahre ihren markanten Niederschlag in der Sanierung und Wiederbelebung des historischen Stadtzentrums von Salvador um den Pelourinho, das Teil des Weltkulturerbes ist.

In musikalischer Hinsicht sind Olodum die Erfinder und prominentesten Vertreter des Samba Reggae, der Anfang der 1980er als Fusion von Samba mit karibischen und afrikanischen Rhythmen entstand. Aus dem Samba Reggae entwickelte sich im Anschluss die popmusikalische Richtung der Axé Music, die ab den 1990ern immer größeren nationalen und internationalen Erfolg erlebte. Olodum und Samba Reggae erfuhren 1990 die erste internationale Aufmerksamkeit, als sie mit Paul Simon den Song "The obvious child" für das Album The Rhythm of the Saints aufnahmen. Darauf folgte 1996 eine Zusammenarbeit mit Michael Jackson für eine Version des Songs “They don't care about us”. Ein Teil des dazugehörigen Videos wurde auf dem Pelourinho in Salvador gedreht (der zweite Drehort war die Favela Dona Marta in Rio de Janeiro) und rückte so die Grupo Cultural Olodum als NRO (Michael Jackson trug in mehreren Szenen ein T-Shirt mit dem prägnanten Logo der Gruppe), afrobrasilianische 
Rhythmen als populärkulturellen Ausdruck und zwei afrobrasilianisch geprägte urbane Szenerien weltweit in den Fokus der Öffentlichkeit.

Antirassismus, afrobrasilianisches Selbstbewusstsein, kulturelle Identität, soziales und politisches Engagement haben so die kulturellen Entwicklungen in Bahia und Salvador in der zweiten Hälfte des 20. Jahrhunderts und bis in die Gegenwart entscheidend mitgeprägt. Neben dem großen Erfolg bahianischer Rhythmen und Percussion-Stile gilt dies auch für die international ähnlich erfolgreiche Tradition der capoeira oder die zunehmende gesellschaftliche Aufwertung und Verbreitung afrobrasilianischer Spiritualität (candomblè).

\section{Recife: revitalisierte Traditionen des Nordostens}

Das etwa 700 km nördlich von Salvador gelegene Recife ist die größte und bedeutendste Metropole des brasilianischen Nordostens. Aufgrund der historischen Besonderheiten dieser Region - insbesondere die langjährige Herrschaft der Niederländer im 17. Jahrhundert - sind im Nordosten bis heute kulturelle Schlüsselelemente verschiedener europäischer Einflüsse in vielfältiger Verschmelzung präsent. Die Volkskultur ist bekannt für ihre Feste und Tänze wie Frevo, Maracatu oder Bumba-meu-boi, in denen europäische, indigene und afrikanische Elemente sich vermischen.

Recife gehörte im 20. Jahrhundert zu den brasilianischen Großstädten, in denen die soziale Segregation und die daraus resultierenden Konflikte am deutlichsten sichtbar wurden. Als Metropole des Nordostens zog die Stadt Migranten aus der gesamten Region an, die sich in Elendssiedlungen entlang der zahlreichen Flussarme und Sumpfgebiete (mangues) niederließen. Vor dem Hintergrund dieser drängenden Probleme mit Armut, Elend, Kriminalität und zunehmender Umweltverschmutzung entstand zu Beginn der 1990er Jahre in Pernambuco mit dem Gravitationszentrum in der "Manguetown" Recife die Bewegung des Manguebeat, die eine musikalische Synthese aus regionalen Musikformen und Folklore (anfangs vor allem Maracatu, später verstärkt auch Forrô), alternativem Rock und digitalen Musiktechnologien vollzog.

Die Pioniere des Manguebeat, die mit nur zwei Alben in rasantem Tempo zunächst in Brasilien und dann auch international auf sich aufmerksam machten, waren Chico Science \& Nação Zumbi. Gegen Ende des Jahrzehnts erweiterte sich das Spektrum musikalischer Mischungen 
und traditioneller Aktualisierungen unter dem Oberbegriff nova cena, die nachhaltig die zeitgenössische Musik Brasiliens zwischen Tradition und Pop/Rock beeinflusste. Ihr bedeutendster Vertreter ist die Band Mundo Livre S/A, die auch schon in den Anfangsjahren des Manguebeat aktiv war.

Ihr Sänger Fred 04 zeichnete gemeinsam mit Chico Science verantwortlich für das 1991 verkündete Manifest der neuen Bewegung, "Caranguejos com Cérebro" (Krebse mit Hirn), das gleich zu Beginn den untrennbaren Zusammenhang von alternativer Kulturszene, Musik, sozialer Bewegung und Umweltschutz unterstreicht. So ging es im Manguebeat neben der Aufwertung und Aktualisierung traditioneller und populärkultureller Elemente des Nordostens immer auch darum, vorrangig junge Menschen für Umweltschutz und soziale Gerechtigkeit im lokalen wie globalen Zusammenhang zu mobilisieren (siehe z. B. das Centro Escola Mangue in Recife: <www.escolamangue.org $>$ ). Symbolträchtig hat man die Bewegung ins Zeichen der Mangrovenwälder gestellt. Dieser komplexe Raum der Biodiversität bildet das die Stadt Recife prägende Ökosystem und ihre sozialen wie kulturellen Lebensadern.

Somit verkörpert die vom Manguebeat ausgehende Entwicklung bis heute eine für den brasilianischen Nordosten charakteristische Verbindung aus neuen Musikformen und sozialer Bewegung im Einsatz für die ethnische, soziale wie kulturelle Anerkennung von Minderheiten. Die darin aktiven Initiativen entwickeln eine Dynamik, die in andere Medien wie Film und Bildende Kunst hineinwirkt, weit über ihre Herkunftsregion hinaus reicht und eine neue Wahrnehmung des Nordostens befördert.

\section{São Paulo: Hip-Hop}

Die Megastadt São Paulo wird häufig auch zu den so genannten global cities gezählt. Sie verkörpert neben ihrer wirtschaftlicher Spitzenposition und kulturellen Avantgarde das gängige Bild vom Großstadtdschungel und urbanen Chaos: eine ausufernde, nicht mehr überschaubare Stadt, permanentes Verkehrschaos, hohe Kriminalität und stark beeinträchtigte Lebensqualität. Die kritische Liebesklärung, die Caetano Veloso 1978 in seinem Song "Sampa" an die "dura poesia concreta de suas esquinas", die harte Poesie des Betons von São Paulo machte, beschreibt diese problematische Dualität sehr treffend. 
In den ersten Jahrzenten des 20. Jahrhunderts betrat São Paulo die brasilianische und bald auch internationale Bühne als erste moderne Metropole des Landes. Literarisch dokumentiert und mythisiert wurde diese urbane Entfesselung der Industrialisierung und des früh schon nicht mehr kontrollierbaren Wachstums 1922 von Mário de Andrade in seiner Paulicéia desvairada. Ein etwas gesetzteres, deswegen aber nicht weniger bewegtes und konfliktbeladenes Bild der Einwandererstadt als Ziel und Hoffnung von Menschen aus Brasilien und Europa zeichnete 1927 Antônio de Alcântara Machado in den Erzählungen von Brás, Bexiga e Barra Funda.

Die Schattenseiten der global city lassen São Paulo als Betonmoloch erscheinen, der unzählige Binnenmigranten anzieht, verschlingt und in seinen Armen- und Elendsvierteln sammelt. In diesem düsteren urbanen Szenario vollzog sich Brasiliens zentrale Entwicklung einer Hip-Hop-Szene, die globale Elemente dieser US-amerikanischen Subkultur mit Aspekten brasilianischer Stadtkulturen vermischte. Grundlegendes musikalisches Merkmal des in São Paulo entstandenen Hip-Hop war in seiner Anfangszeit in den 1990er Jahren die Repräsentation der rauen Wirklichkeit und des Überlebenskampfes in der Megastadt durch harte Beats und aggressive Texte. Die Pioniere des brasilianischen Hip-Hop sind die Racionais MCs, die mit Alben wie Holocausto urbano (1990) und Sobrevivendo no inferno (1997) Meilensteine der brasilianischen Musikgeschichte geschaffen haben und gleichzeitig unerwarteten kommerziellen Erfolg erzielten. Die beiden Titel bilden Anspielungen auf die ursprüngliche Lebenswirklichkeit der Gruppenmitglieder im Favelakomplex von Capão Redondo im Südwesten São Paulos. In ihren Texten und ihrem außermusikalischen Engagement klagen die Racionais MCs die allgemeine Marginalisierung und Diskriminierung der armen und mehrheitlich dunkelhäutigen Menschen in der Peripherie an, denunzieren Missstände in staatlichen Erziehungseinrichtungen oder Jugendgefängnissen, Polizeigewalt und Perspektivlosigkeit. Wie mittlerweile auf der ganzen Welt bildet Hip-Hop als paradigmatische urbane Gegenkultur eine Plattform, um über die Musik hinaus mit Kulturaktivitäten wie Breakdance oder Graffiti, Drogen- und Kriminalitätsprävention oder Ausbildungsangeboten gesellschaftliche Veränderungen zu bewirken.

Im Schoß der Hip-Hop-Kultur vollzog sich auch der Übergang von den Rap-Texten über die Lyrik hin zur Literatur im Allgemeinen. Der bekannteste Vertreter dieser unter dem Label der "literatura marginal" sich etablierenden Literatur ist Ferréz, der ebenfalls in Capão Redondo auf- 
gewachsen ist und diese unmittelbare Lebenswirklichkeit - die Ausweglosigkeit, Diskriminierung und Selbstzerstörung junger Erwachsener in São Paulos Peripherie zwischen schnellem Geld und Ruhm, Drogen und Gewalt - in seinen ästhetisch mit dem Rap verbundenen Romanen (Capão Pecado, 2000, und Manual prático do ódio, 2003) schonungslos und kritisch zur Darstellung bringt. Ferréz hat Capão Redondo bewusst nicht verlassen und nutzt dort seine Bekanntheit, um Nachwuchsautoren der "literatura marginal" unter anderem mit einem eigenen Verlag (Selo Povo) zu fördern und die Hip-Hop-Szene, insbesondere mit einem eigenen Modelabel (1DASUL), weiter mitzugestalten.

\section{Rio de Janeiro: neue städtische Bewegungen}

Die Favelas oder comunidades - im Sinne einer selbstbewussten Selbstbenennung ihrer Bewohner und Aktivisten - sind seit der 'Afrikanisierung des Karnevals' im Übergang vom 19. zum 20. Jahrhundert unverzichtbare Grundlage und Nährboden für diesen heute international vermarkteten Wettbewerb, den sie in Rio de Janeiro mit ihren Sambaschulen, Umzügen und samba enredos bestreiten. Auch so typische Musikformen Rio de Janeiros wie Samba und Choro/Chorinho sind lebendiger Bestandteil des kulturellen Lebens der comunidades, wo sie mit neuen musikalischen und jugendkulturellen Formen wie dem seit den 1970er Jahren entstandenen und seit den 1990ern auch international beachteten funk in Dialog und Konkurrenz stehen.

Regulierung und Formalisierung beeinflussen diesen kulturellen Bereich dahingehend, dass die Stadtregierung den Sambaschulen der oberen Ligen seit 2005 in der so genannten "Cidade do Samba" (der "Stadt des Samba") jeweils eigene Hallen für ihre Umzugsvorbereitungen zur Verfügung stellt. Die Sambaschulen können so eine bessere Infrastruktur nutzen und interessierten Besucher wird ein 'geordnetes' Umfeld garantiert, das auch nicht innerhalb einer Favela liegt. Doch die konkrete Einbettung und damit Verankerung der Sambaschulen in ihren Vierteln, Favelas und Gemeinschaften wird dadurch geschwächt. Allgemein spiegeln die Widersprüche des Karnevals von Rio de Janeiro viele der Probleme, die den in der Stadt herrschenden Konflikten zugrunde liegen. Die weiter voranschreitende Kommerzialisierung des Großevents steigert die Spannung und Kluft zwischen Leitungsgremien und Basis, hinzu kommt der 
Einfluss der Drogenbanden, die sich seit den 1990er Jahren zunehmend als wichtigste 'Sponsoren' der Sambaschulen betätigen.

Die wachsende Anerkennung für die Ursprünge solch bedeutender Kulturgüter steht in enger Wechselbeziehung zu einem strukturellen Wandel ihres Entstehungskontextes. Der Erfolg kultureller Manifestation und Initiativen beförderte die zivilgesellschaftliche und politische Repräsentation der comunidades, wurde von ebendieser aber auch erst in seiner aktuellen Form ermöglicht. Neue soziale Akteure haben in jüngerer Vergangenheit die nationale und internationale Bühne betreten. Heute bemühen sich NROs und kulturelle Initiativen in den armen Stadtvierteln und Favelas, wie zum Beispiel das Zentrum für Kooperation und Bewohneraktivitäten (Centro de Cooperação e Atividades Populares) im Favelakomplex von Manguinhos, um neue Formen des empowerment und der nachhaltigen lokalen Entwicklung für die arme und einfache Bevölkerung mit ihren ökonomischen, sozialen und kulturellen Bedürfnissen. Gerade im Zuge der digitalen Revolution der Medien und des Internets schaffen diese Organisationen immer stärker präsente Kanäle für ihre eigenen Stimmen und Bilder, welche in großem Maße über eigene Kulturgüter wie Musik und Tanz, Journalismus und Film/Videokunst transportiert werden ( $<$ http://www. vivafavela.com.br $>$ ). Auch bereits erfolgreiche Musiker, Schriftsteller oder Künstler engagieren sich dafür, ihre comunidades durch die Gründung solcher kultureller und sozialer Institutionen zu unterstützen. Insgesamt bietet sich ein Bild neu gestalteter Kanäle der Kommunikation und Interaktion zwischen den armen 'Inseln' der fragmentierten Großstädte.

Soziale Bewegungen und Zivilgesellschaft greifen hier ineinander und treten in Wechselwirkung mit kulturellen Entwicklungen in den comunidades, die weit über den Bereich der 'informellen Stadt' hinaus wirken. Die Grupo Cultural AfroReggae (<www.afroreggae.org.br>) wurde 1993 in Reaktion auf eine Reihe von Massakern an unschuldigen Favela-Bewohnern durch korrupte Polizisten gegründet - diese Entstehungsgeschichte wird in dem Dokumentarfilm Favela Rising von Jeff Zimbalist und Matt Mochary (2005) sehr anschaulich erzählt. Heute kann AfroReggae auf eine beeindruckende Vielfalt an sozialen Projekten in der Jugendarbeit verweisen. Neben national wie international erfolgreichen Musikgruppen mit eigener Produktionsfirma widmet sich die NRO der Medienarbeit, betreibt ein Mode-Label und eine Zirkusschule, leistet medizinische Aufklärung und Hilfe unter den Bewohnern und kann eigene Schulstipendien vergeben. 
Ein weiteres Beispiel bildet die Kooperative CoopaRoca (<www.coopa-roca.org.br>) aus Rios größter comunidade Rocinha, die mit ihren Modekreationen aus Resten und Abfall in die Geschäfte der wohlhabenden Zona Sul einzog. Dieses frühe Beispiel von 'favela chic' hat Schule gemacht und so wirkt z. B. im Umfeld von AfroReggae und deren internationaler Kooperation mit britischen NROs das Kollektiv Retalhos Cariocas (<www.retalhoscariocas.com>) mit einem ähnlichen Modell von Design, Produktion und Vertrieb in Eigenverantwortung sowie Arbeits- und Ausbildungsmöglichkeiten für die Gemeinschaft.

Das in Brasilien und im Ausland ebenso euphorisch wie kritisch rezipierte Filmprojekt Cidade de Deus von Fernando Meirelles und Kátia Lund (2002) ist ein gutes Beispiel für die Konflikte und Probleme, die andererseits gerade aus dem Erfolg solcher sozialer und kultureller Projekte entstehen können. Das dem Film zugrunde liegende Buch gleichen Titels von Paulo Lins (1997) war ursprünglich ein stadtanthropologisches Forschungsprojekt in den etwa 120.000 Einwohner umfassenden comunidades, die sich um den 1962-1965 als stadtplanerisches Projekt implantierten Wohnkomplex "Cidade de Deus" angesiedelt haben. Statt einer wissenschaftlich-engagierten Studie entstand schließlich ein literarisches Projekt über drei Generationen von Drogenbossen, das einen überraschenden Publikumserfolg erzielte und umfassende Diskussionen im Spannungsfeld von Ästhetik, Gesellschaft und Politik anstieß. Für die Dreharbeiten zur Verfilmung an Originalschauplätzen und mit zahlreichen lokalen Laiendarstellern wurden Filmproduktion und soziale Arbeit in den comunidades eng miteinander verzahnt, es entstanden 'Nebenprojekte' wie der Dokumentarfilm Notícias de uma guerra particular (1999) über die Hintergründe der Gewaltspirale in Rios Favelas, die erfolgreiche, gemeinsam mit TV Globo produzierte Fernsehserie Cidade dos Homens (2002-05) und der gleichnamige Kinofilm (2007), in denen zumindest teilweise auch das Lebens der favelados jenseits von Drogengewalt und Kriminalität einem breiten Publikum vorgestellt wurden.

Kritik wurde vor allem gegen die international erfolgreiche und auf ihre ästhetisierenden Dimensionen reduzierte Darstellung der Favelas als exotischer Freiraum sowie der Gewalt als deren Hauptmerkmal vorgebracht, wie sie sich im dem vor allem kommerziell eingesetzten Label $f a$ vela fiction niederschlägt. Filme wie die beiden Teile von Tropa de elite (Elite squad, 2007 und 2010, Regie: José Padilha) werden einerseits diesem Etikett zugeordnet und für ihre als übertrieben empfundene Gewaltdarstellung kritisiert, andererseits schließen sie an die von Cidade de Deus/City of 
God initiierte Entwicklung an, die Schattenseiten der brasilianischen Stadtlandschaften im Allgemeinen sowie der 'wunderbaren Stadt' Rio de Janeiro im Besonderen unverstellt vor Augen zu führen und auch die Ursachen dieser Situation - bei Tropa de elite vor allem die den gesamten Polizei- und Staatsapparat durchsetzende Korruption - zu denunzieren.

Im Vorfeld der Fußball-WM 2014 und der Olympischen Spiele 2016

Schon seit langen Jahrzehnten versucht der brasilianische Staat, durch entsprechende Maßnahmen und mit mal größerem, mal geringerem Erfolg, mit den urbanen Peripherien in Dialog zu treten. Ein zentraler Ansatz ist dabei immer wieder die Schaffung neuer bzw. die Wiederbelebung degradierter alter Räume - wie den historischen Stadtzentren - als 'kulturelle Territorien', die wie ein Hebel für eine allgemeine soziale Verbesserung angrenzender Stadtbereiche wirken könnten.

Noch älter und problematischer ist der Ansatz staatlicher Intervention, über die Regulierung und Formalisierung der Armenviertel (community upgrading) das Problem der sozialen Konflikte sowie der mit ihnen einhergehenden Kriminalität und Gewalt einzudämmen. In Rio de Janeiro etwa wurde dies mit dem 1993 von der Stadtverwaltung begonnenen Programm Favela-Bairro unternommen, in dessen Zuge stadtplanerische Entwürfe mehr oder weniger im Dialog mit Organisationen der betroffenen comunidades die Favelas mit ihrem charakteristischen - und als eigene Ästhetik begreifbaren - Chaos aus spontanen Bauten und verwinkelten Straßenzügen mit Kanalisation, Stromversorgung und Asphaltierung ausstatten und dort neue öffentliche Räume schaffen wollten.

Nicht zuletzt im Hinblick auf die anstehenden Großereignisse in der Stadt wurden bisherige Upgrading-Programme 2010 gebündelt und unter dem Namen "Morar carioca" (Wohnen in Rio) neu aufgelegt. Im Zuge dieses ambitionierten Ansatzes sollen bis 2020 über 1.000 Favelas in die Stadtstruktur integriert werden. Überhaupt erfahren alle staatlichen Initiativen im Zusammenhang mit sozialen Fragen in Rio de Janeiro durch die Vorbereitung auf die Fußballweltmeisterschaft 2014 und die Olympischen Spiele 2016 neue Impulse bzw. stehen sie nun unter deren Vorzeichen. Das offizielle Olympia-Portal der Stadtverwaltung von Rio de Janeiro bietet einen sehr guten Überblick über die verschiedenen Bereiche und einzelnen Projekte (<www.cidadeolimpica.com.br>). 
Große mediale Aufmerksamkeit erfuhr z. B. die 2011 eröffnete Seilbahn (die sog. "Teleférico do Alemão"), die sich mit sechs Stationen auf etwa 3,5 km Länge über die riesige Favela "Complexo do Alemão" erstreckt und für die Bewohner als Nahverkehrsmittel dient. Gleichzeitig soll die Seilbahn zu einer Attraktion für brasilianische wie internationale Touristen werden und so weiter reichende Strukturveränderungen in der Region bewirken. Im Juni 2012 wurde in Rios Nordzone die Parkanlage "Parque de Madureira" eröffnet, die mit ihrem Angebot für Freizeitaktivitäten und Sport einen weit reichenden stadtplanerischen Eingriff in dieser Region bedeutet. Im Zusammenspiel mit der ebenfalls dort geplanten Schnellbuslinie "Transcarioca" soll eine strukturelle Aufwertung befördert werden. Die Schaffung neuer Schnellbuslinien quer durch Rio de Janeiro, um vor allem auch die Peripherie an die bürgerliche Infrastruktur anzuschließen, bildet dabei sicher das Herzstück der vorbereitenden Maßnahmen für die Fußball-WM 2014 und die Olympischen Spiele 2016 in Rio de Janeiro. Die drei Expresslinien sollen die Struktur des öffentlichen Nahverkehrs insgesamt verbessern und damit vor allem signifikante Erleichterungen für die Mobilität der Bewohner bringen, die auf öffentliche Verkehrsmittel angewiesen sind oder auf das Auto verzichten wollen.

Die Kehrseite dieser dialogischen Ansätze bilden die eingangs erwähnten Militäraktionen zur 'Säuberung' der Favelas von den dort herrschenden Drogenbanden. Diese Strategie ist ebenso alt wie die Favelas selbst, schon zu Beginn des 20. Jahrhunderts versuchte der Staat, den 'Makel' der illegalen Elendsviertel durch Zwangsräumung bzw. Umsiedlung der Bewohner zu beseitigen. Auch wurden z. B. unter der Militärregierung in den 1960er und 1970er Jahren Projekte zur Zerstörung von Favelas mit der Umsiedlung ihrer Bewohner in Mustersiedlungen durchgeführt. Beides allerdings nicht mit dem beabsichtigten Erfolg. Der bereits erwähnte Komplex der Cidade de Deus entstand gerade aus einer solchen Mustersiedlung heraus.

Neu ist im Vorfeld der sportlichen Megaevents in den 2010er Jahren der Versuch, nach der Vertreibung der Drogenbanden die staatliche Ordnung durch speziell geschulte Polizeieinheiten in 'Friedensmission' (die UPPs) zu etablieren und zu stabilisieren. Einerseits bedeutete die Schaffung dieser neuartigen Polizeieinheiten eine Abkehr von der frontalen und unnachgiebigen Konfrontation mit den Drogenbanden, vor allem auch von der pauschalen Kriminalisierung der comunidades. Auf der anderen Seite jedoch übernehmen die UPPs in der Regel Sicherungsaufgaben in 
Favelas, die zuvor vom Militär in massiven bewaffneten Aktionen regelrecht von den kontrollierenden Drogenbanden 'befreit' oder 'gesäubert' wurden. Neben dem Vorwurf der staatlichen Bevormundung und getarnten Kontrolle der comunidades durch die 'Friedenspolizei' beinhaltet eine häufig vorgebrachte Kritik an dieser neuen Strategie die Befürchtung, dass es sich dabei letzten Endes nur um eine medienwirksam inszenierte 'Befriedung' der urbanen Brennpunkte im Vorfeld der international bedeutsamen Großereignisse handelt, die nach dem Ende der Olympischen Spiele 2016 bald wieder sich selbst überlassen werden.

\section{Schlussbemerkung}

Räume der Armut und Informalität sind nicht nur Herde von Gewalt, Drogen, Verbrechen und anderen Bedrohungen für die bürgerliche Stadt, auch wenn sie in der allgemeinen gesellschaftlichen und internationalen Wahrnehmung oftmals als solche erscheinen. Sie sind vor allem auch Räume lebendiger kultureller Traditionen und Neuschöpfungen, die sich oft kritisch mit den Erfordernissen und Beschränkungen ihres Entstehungskontextes auseinandersetzen. Diese kulturellen Dynamiken wirken produktiv und kreativ auf ihr Umfeld ein, verwandeln sich in soziale und politische Projekte oder verbinden sich mit den analog zu ihnen entstehenden 'städtischen Bewegungen', um gemeinsam ihren marginalisierten Kulturformen und Gesellschaftsgruppen ein Gesicht, eine Stimme, einen Diskurs zu geben und ihnen in der gesamtgesellschaftlichen Diskussion Gewicht zu verleihen: Samba, Karneval, Funk oder Hip-Hop, blocos afro oder Manguebeat sind sehr unterschiedliche kulturelle Phänomene und bewirken nicht unmittelbar den gesellschaftlichen Wandel, streben ihn teilweise nicht einmal an. Aber sie sind Ausdruck von Dynamiken und Transformationen innerhalb bestimmter Gesellschaftsgruppen und Teil einer globalen Entwicklung, in der populäre Kulturen, Medien und neue Formen des Engagements immer größere Bedeutung und vielfältige Wirkungen entfalten.

Besonders interessant ist hierbei zu beobachten, wie solche lokalen Initiativen über neue Medien und im Kontext der Globalisierung 'von unten' verstärkt neuartige Formen der internationalen Vernetzung aufbauen. Diese Entwicklung führt zur zunehmenden Zusammenarbeit brasilianischer NROs und Projekte mit Partnern auf der ganzen Welt, einer- 
seits in den Industrieländern, ebenso aber im 'globalen Süden', wie das Beispiel der Grupo Cultural AfroReggae auf beeindruckende Weise zeigt. Dadurch entsteht eine neue weltweite Sichtbarkeit und Anerkennung dieser lokalen Aktivitäten, die in dieser Form in Brasilien selbst - seitens der bürgerlichen Stadt - meist gar nicht gegeben ist. Ähnlich verhält es sich mit der Anerkennung und dem Erfolg des in Brasilien weiterhin stark 'ghettoisierten' funk, der unter dem Label Rio funk oder Favela funk mittlerweile Einzug in die Clubs der Welt gehalten hat und außerhalb Brasiliens bereits als neue Ausdrucksform brasilianischer Populärkultur anerkannt wird. Allgemein ist so festzustellen, dass weite Teile dieser Akteure brasilianischer Stadtkulturen und neuen sozialen Bewegungen in ihrem unmittelbaren Stadtraum zwar weiterhin marginalisiert sind und um allgemeingesellschaftliche Anerkennung ringen müssen, auf globaler Ebene dagegen diese Isolation bereits haben überwinden können.

\section{Literaturhinweise}

Caldeira, Teresa Pires do Rio (2000): Cidade de muros. Crime, segregação e cidadania em São Paulo. São Paulo: Editora 34.

Castro, Mary Garcia/Abramovay, Miriam/Rua, Maria das Graças et al. (Hg.) (2001): Cultivando vida, desarmando violências. Experiências em educação, cultura, laz̧er, esporte e cidadania com jovens em situação de pobręa. Brasília: UNESCO/Fundação Kellogg.

GouvêA, Ronaldo Guimarães (2005): A questão metropolitana no Brasil. Rio de Janeiro: Editora FGV.

Herschmann, Micael (Hg.) (1997): Abalando os anos 90. Funk e Hip-bop. Globalização, violência e estilo cultural. Rio de Janeiro: Rocco.

LANz, Stephan (Hg.) (2004): City of COOP. Ersatzökonomien und städtische Bewegungen in Rio de Janeiro und Buenos Aires. Berlin: b_books.

Markman, Rejane Sá (2007): Música e simbolização. Manguebeat: contracultra em versão cabocla. São Paulo: Annablume.

Moura, Roberto (1995): Tia Ciata e a Pequena África no Rio de Janeiro. 2. Aufl. Rio de Janeiro: Prefeitura da Cidade do Rio de Janeiro/Secretaria Municipal da Cultura.

Neat, Patrick/Platt, Damian (2010): Culture Is Our Weapon. Making Music and Changing Lives in Rio de Janeiro. London: Penguin.

Santos, Milton (1993): A urbaniz̧ação brasileira. São Paulo: Hucitec.

Schaun, Angela (2002): Prácticas educomunicativas. Grupos afro-descendentes Salvador - Babia, Ara Ketu, Ilê Aiyê, Olodum, Pracatum. Rio de Janeiro: Mauad. 